\title{
Stability Indicating RP-HPLC Method for the Simultaneous Estimation of Metformin Hydrochloride, Pioglitazone Hydrochloride and Glibenclamide in Bulk and Pharmaceutical Dosage Forms
}

\author{
SaiThanuja V, Chandan R S*, Anandkumar R Tengli, Gurupadayya B M, \\ Prathyusha W \\ Department of Pharmaceutical Chemistry, JSS College of Pharmacy, JSS University, Sri Shivarathreeshwara \\ Nagar, Mysore-570015, India
}

\begin{abstract}
A simple, selective and precise Stability indicating RP-HPLC method was developed for the simultaneous estimation of Metformin Hydrochloride, Pioglitazone Hydrochloride and Glibenclamide in the Bulk and Pharmaceutical Dosage Forms using glimepiride as an internal standard. The chromatographic separation of the three drugs was achieved on a reverse phase Inertsil-ODS, C18, $100 \mathrm{X} 4.6 \mathrm{~mm}$, $5 \mu \mathrm{m}$ column using $0.1 \mathrm{M}$ Ammonium acetate buffer ( $\mathrm{pH}$ 4.5adjusted by using formic acid) and Acetonitrile in the ratio of $45: 55 \mathrm{v} / \mathrm{v}$ with flow rate of $0.8 \mathrm{ml} / \mathrm{min}$ with injection volume $20 \mu \mathrm{L}$ and the detection was carried out at $254 \mathrm{~nm}$. The retention time of metformin hydrochloride, pioglitazone hydrochloride and glibenclamide and glimepiride were found to be 1.1, 4.5, 5.9, 6.5min respectively. The drug products were subjected to stress conditions of acidic, alkaline, oxidation, UV and Thermal conditions. The degradation products were well resolved from Metformin Hydrochloride, Pioglitazone Hydrochloride and Glibenclamide peaks, thus indicating the stabilityindicating nature of the method. The linear regression analysis data for the calibration plots showed good linear relationship in the concentration range of 62.5-375.00 $\mathrm{g} / \mathrm{ml}$ for metformin hydrochloride, 3.75-22.5 $\mu \mathrm{g} / \mathrm{ml}$ for pioglitazone hydrochloride and $1.25-7.50 \mu \mathrm{g} / \mathrm{ml}$ for glibenclamide. The developed method was successfully validated in accordance to ICH guidelines. Hence, this method can be conveniently adopted for the routine analysis in quality control laboratories.
\end{abstract}

Key words: Metformin Hydrochloride, Pioglitazone Hydrochloride, Glibenclamide, RP-HPLC

\section{Introduction}

Metformin $\mathrm{HCl}$ (MET) is an anti diabetic drug. Chemically, it is 1,1-dimethyl biguanide hydrochloride (Fig. 1). Metformin decrease the gluconeogenisis and increases the glucose uptake by muscles and fat cells. It is indicated for the treatment of type II diabetes mellitus, used alone or in combination with sulfonylurea's, alphaglycosidase inhibitors, or insulin.

Pioglitazone (PIO) is a thiazolidinedione antidiabetic agent, chemically it is (RS)-5-(4-[2(5ethylpyridin 2-yl) ethoxy] benzyl) thiazolidine-2,4-dione (Fig. 2). It selectively stimulates the peroxisome proliferator-activated receptor gamma (PPAR- $\gamma$ ) and to a lesser extent PPAR- $\alpha$. It is used for the treatment of type II diabetes mellitus either alone or in combination with other oral anti diabetic drugs.

Glibenclamide (GLI) is a second generation sulphonyl urea oral hypoglycemic agent, chemically it is 1-[4-[2-(chloro 2-methoxybenzamido) ethyl]-benzene sulphonyl]-3cyclo hexyl urea, 5-chloro-N-[2-[4[[[(cyclohexyl (amino) carbonyl]-amino] sulphonyl] phenyl] ethyl-2- methoxy benzamide (Fig. 3). It is used to assist to control mild to moderately severe type II diabetes mellitus that does not require insulin that can be adequately controlled by diet alone [1].

Literature survey revealed HPLC, RP-HPLC, LC-MS, Spectrofluorimetric and simultaneous UV spectrophotometric methods are reported for the estimation of metformin hydrochloride [2-5], pioglitazone hydrochloride [6-9] and glibenclamide [10] alone or in combination with other anti-diabetic agents. So the present study aim to develop a simple, selective and precise RP-HPLC method for the simultaneous estimation of MET, PIO and GLI in bulk drug samples and in combined dosage formulation.

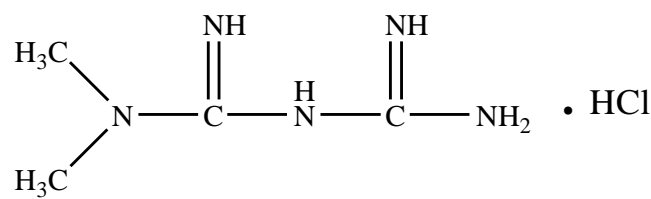

Fig. 1 Structure of Metformin $\mathrm{HCl}$ 
<smiles>CCc1ccc(CCOc2ccc(CC3SC(=O)NC3=O)cc2)nc1</smiles>

Fig. 2 Structure of Pioglitazone HCl<smiles>COc1ccc(Cl)cc1C(=O)NCCc1ccc(S(=O)(=O)NC(=O)NC2CCCCC2)cc1</smiles>

Fig. 3 Structure of Glibenclamide

\subsection{Chromatographic Conditions}

\section{Materials And Methods}

The WATERS HPLC with PDA detector and Empower2 software was employed for the present study. The chromatography determination performed at ambient temperature by using Inertsil $\mathrm{BDS}_{18}(100 \times 4.6,5 \mu \mathrm{m})$ column, with a mobile phase composed of mixture of $0.1 \mathrm{~N}$ ammonium acetate buffer of $\mathrm{pH} 4.5$ and Acetonitrile in the ratio of 45:55. The chromatography run time was maintained up to 10.0 min with flow rate at $0.8 \mathrm{~mL} / \mathrm{min}$ with injection volume $20 \mu \mathrm{L}$ and the eluent was monitored at $254 \mathrm{~nm}$.

\subsection{Reference Standards, Reagents}

Working standards, Metformin, Glibenclamide, Pioglitazone and Glimepiride was obtained from Ranbaxy laboratories, New Delhi, India. Acetonitrile and water employed for the preparation of mobile phase were of HPLC grade was obtained from Merck limited, Mumbai. The pharmaceutical dosage form containing $500 \mathrm{mg}$ MET, $15 \mathrm{mg}$ PIOG and $5 \mathrm{mg}$ GLIB, Triglycomet $520 \mathrm{mg}$, 20 tablets (USV Pharmaceuticals Ltd.) purchased from a local drug store.

\subsection{Preparations of standard solution}

$500 \mathrm{mg}$ of metformin hydrochloride, $15 \mathrm{mg}$ of pioglitazone hydrochloride and $5 \mathrm{mg}$ of glibenclamide were weighed and transferred into a $100 \mathrm{ml}$ volumetric flask and $50 \mathrm{ml}$ of diluents was added. This solution was sonicated to dissolve and final volume was made with diluent. $10 \mathrm{ml}$ of the above solution was transferred into the $100 \mathrm{ml}$ volumetric flask and diluted to final volume with diluent.

\subsection{Sample preparation}

20 tablets were weighed and finely powdered. Sample quantitatively equivalent to $500 \mathrm{mg}$ Metformin, $15 \mathrm{mg}$ of Pioglitazone and $5 \mathrm{mg}$ of glibenclamide was transferred in to $100 \mathrm{~mL}$ volumetric flask and $50 \mathrm{ml}$ of diluent was added, sonicated to dissolve the sample for 10 minutes and dilute to volume with diluent. Further the solution was filtered through $0.45 \mu$ membrane filter and $10 \mathrm{ml}$ of this solution was diluted to $100 \mathrm{ml}$ with diluent.

\subsection{Assay procedure}

In case of marketed formulations, twenty tablets were taken and finely powdered and an accurate amount of powder was transferred into a $100 \mathrm{ml}$ volumetric flask. The stock solution was further diluted with mobile phase. The column was equilibrated atleast $30 \mathrm{~min}$, with the mobile phase flowing through the system with a flow rate of $0.8 \mathrm{ml} / \mathrm{min}$ and detector was set at a wavelength of $254 \mathrm{~nm}$. The retention times of metformin hydrochloride, pioglitazone hydrochloride, gilbenclamide and internal standard glimepiride in bulk drug were found to be 1.1, 4.5 5.9 and 6.5 mins (Fig. 4) and the retention times of metformin hydrochloride, pioglitazone hydrochloride, gilbenclamide and internal standard glimepiride were found to be 1.1, 4.6, 5.9 and 6.5 mins (Fig. 5). Blank chromatogram is shown (Fig. 6). The \% purity of metformin hydrochloride, pioglitazone hydrochloride and glibenclamide in tablet dosage form were compiled and reported in Table1. 
Stability Indicating RP-HPLC Method for the Simultaneous Estimation of Metformin Hydrochloride,

Table: 1 Determination of MET, PIO and GLI in Tablet dosage form

\begin{tabular}{|l|c|c|c|}
\hline \multicolumn{1}{|c|}{ Drug } & Label claim (mg) & Amount found (mg) & Drug Content (\%) \\
\hline Metformin hydrochloride & 500.0 & 498.33 & 99.67 \\
\hline Glibenclamide & 5.02 & 5.02 & 100.48 \\
\hline Pioglitazone hydrochloride & 15.15 & 15.15 & 101.00 \\
\hline
\end{tabular}

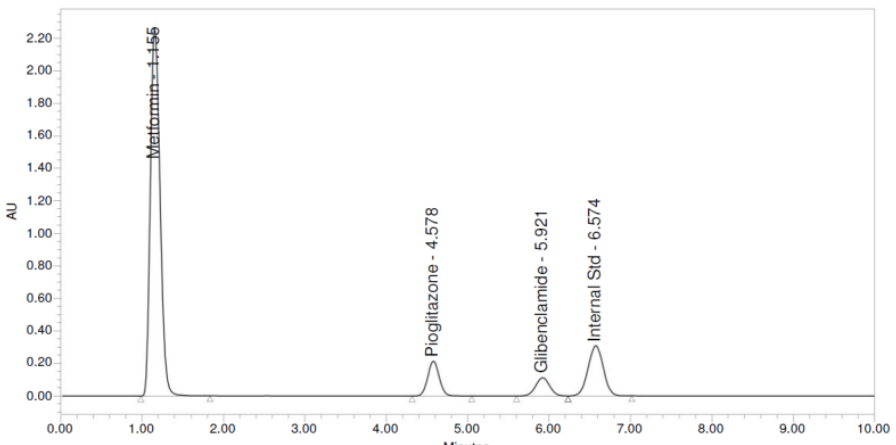

Fig. 4 Standard chromatogram of metformin hydrochloride, pioglitazone, gilbenclamide and internal standard glimepiride

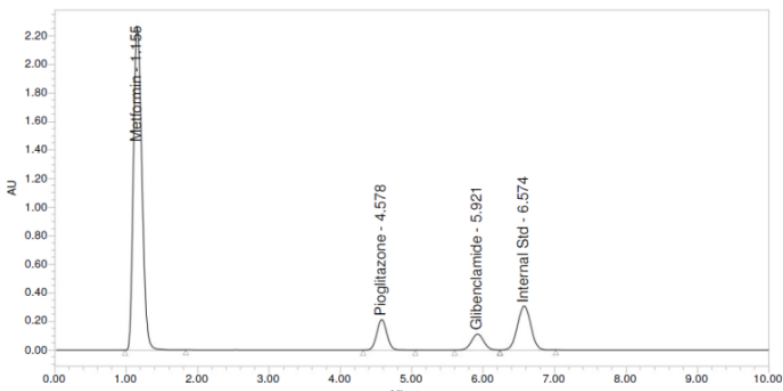

Fig. 5 Sample chromatogram of metformin hydrochloride, pioglitazone, gilbenclamide and internal standard glimepiride

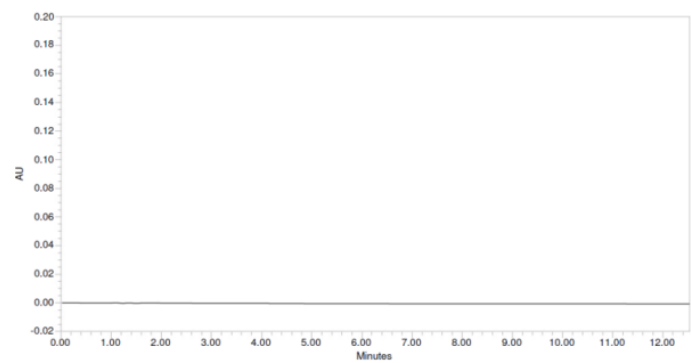

Fig. 6 Chromatogram of blank

\section{Validation}

After the method conditions were established as described above, method was validated as per ICH guidelines. ${ }^{[12-14]}$ The accuracy, precision, Linearity, limit of detection (LOD) and quantification (LOQ) were determined.

\subsection{Linearity}

The linearity of the method was established by preparing the series of dilutions from the standard stock mixture to get the concentrations of metformin hydrochloride $(62.5-375.00 \mu \mathrm{g} / \mathrm{ml})$ pioglitazone hydrochloride $(3.75-22.5 \mu \mathrm{g} / \mathrm{ml})$ and $(1.25-7.50 \mu \mathrm{g} / \mathrm{ml})$ for glibenclamide and the above solutions were injected into the HPLC system. The standard Calibration curve for metformin hydrochloride (Fig. 7), pioglitazone hydrochloride (Fig. 8) and glibenclamide (Fig. 9) was constructed by plotting their response ratios (ratios of the peak area of the analytes) against their respective concentrations. Linear regression was applied and Slope (a), intercept (b), correlation coefficient (r) was determined. The values are summarized in Table 2. 
Stability Indicating RP-HPLC Method for the Simultaneous Estimation of Metformin Hydrochloride,

Table 2: Linearity

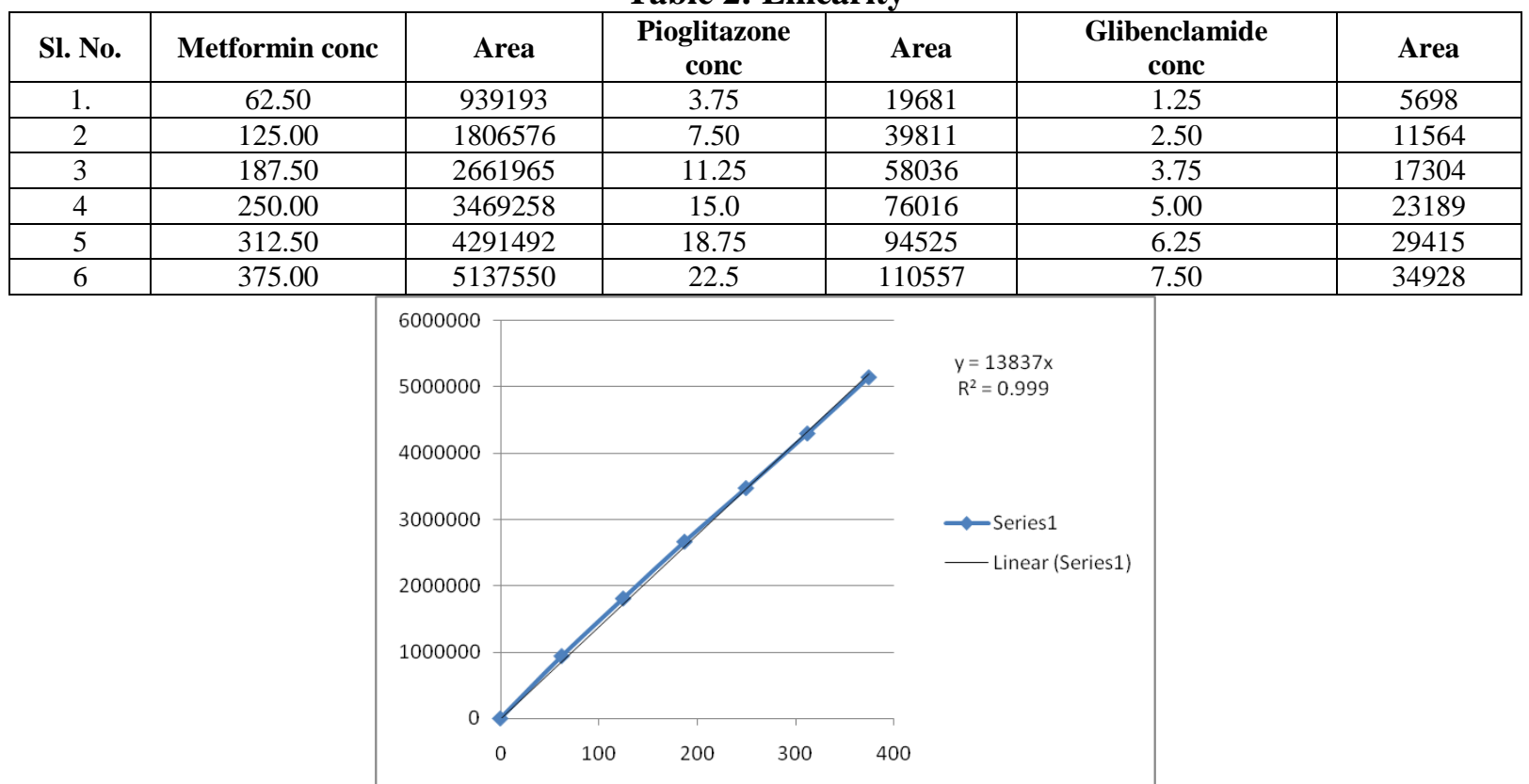

Fig. 7 Standard Calibration Graph of Metformin Hydrochloride

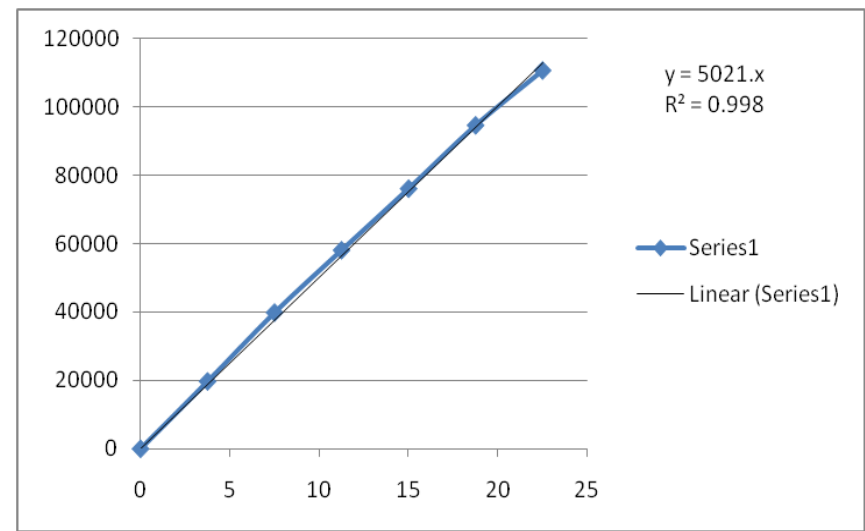

Fig. 8 Standard Calibration Graph of Pioglitazone Hydrochloride

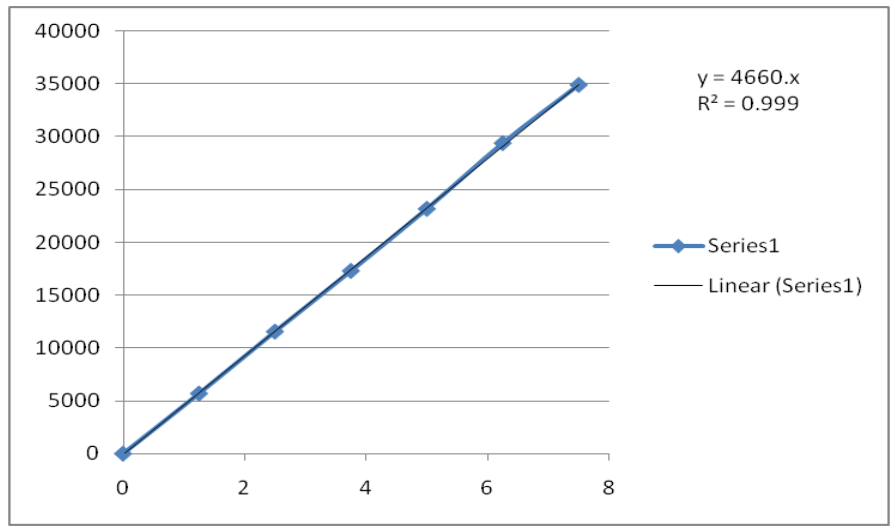

Fig. 9 Standard Calibration Graph of Glibenclamide

\subsection{Accuracy}

Accuracy was determined in terms of percentage recovery. Sample solution spiked with the analytes at three different concentration levels 62.5-375.0 $\mu \mathrm{g} / \mathrm{ml}$ of metformin hydrochloride,3.75-22.5 $\mu \mathrm{g} / \mathrm{ml}$ of pioglitazone hydrochloride and $1.25-7.50 \mu \mathrm{g} / \mathrm{ml}$ of glibenclamide. Another set of standard mixtures at the same 
concentration levels was also prepared with the diluents. Sample and standard solutions are injected into the HPLC system in triplicate. Percentage recoveries of metformin hydrochloride, pioglitazone hydrochloride and glibenclamide were calculated. The values are summarized in Table 3 .

Table 3: Recovery studies

\begin{tabular}{|l|c|c|c|c|c|}
\hline \multicolumn{1}{|c|}{ Drug } & Conc & $\begin{array}{c}\text { Peak area } \\
\text { (avg) }\end{array}$ & $\begin{array}{c}\text { Amount of drug } \\
\text { added }\end{array}$ & $\begin{array}{c}\text { Amount of drug } \\
\text { found }\end{array}$ & \% recovery \\
\hline \multirow{3}{*}{$\begin{array}{l}\text { Metformin } \\
\text { hydrochloride }\end{array}$} & $80 \%$ & 2766617 & 200.01 & 200.97 & 100.48 \\
\cline { 2 - 6 } & $100 \%$ & 3428658 & 250.00 & 249.06 & 99.62 \\
\hline \multirow{3}{*}{$\begin{array}{l}\text { Pioglitazone } \\
\text { hydrochloride }\end{array}$} & $120 \%$ & 4136170 & 300.05 & 300.46 & 100.14 \\
\cline { 2 - 6 } & $100 \%$ & 52908 & 12.00 & 11.99 & 99.91 \\
\hline \multirow{3}{*}{ Glibenclamide } & $120 \%$ & 63434 & 15.00 & 15.06 & 100.4 \\
\cline { 2 - 6 } & $80 \%$ & 18488 & 4.00 & 18.06 & 100.21 \\
\cline { 2 - 6 } & $100 \%$ & 23169 & 5.00 & 3.99 & 99.72 \\
\hline
\end{tabular}

\subsection{Precision}

Method precision was determined both in terms of repeatability (injection and analysis) and intermediate precision (intra-day and inter-days reproducibility). In order to determine injection repeatability, samples spiked with metformin hydrochloride, pioglitazone hydrochloride and glibenclamide were injected 6 times into HPLC system and repeatability of the retention time and peak area were determined and expressed as mean and \%RSD calculated from the data obtained. The values are summarized in Table 4, 5, 6 and 7.

Table: 4 System Precision

\begin{tabular}{|c|c|c|c|c|c|c|}
\hline \multirow{2}{*}{ Name } & \multicolumn{2}{|c|}{ Metformin } & \multicolumn{2}{c|}{ Pioglitazone } & \multicolumn{2}{c|}{ Glibenclamide } \\
\cline { 2 - 7 } & RT & Area & RT & Area & RT & Area \\
\hline System Precision-1 & 1.153 & 3390269 & 4.598 & 50264 & 5.999 & 22594 \\
\hline System Precision-2 & 1.152 & 3391873 & 4.588 & 51645 & 5.997 & 22789 \\
\hline System Precision-3 & 1.151 & 3402394 & 4.590 & 50611 & 5.996 & 22738 \\
\hline System Precision-4 & 1.152 & 3396645 & 4.591 & 51444 & 5.997 & 22765 \\
\hline System Precision-5 & 1.154 & 3398545 & 4.587 & 50244 & 5.995 & 22884 \\
\hline System Precision-6 & 1.152 & 3395773 & 4.586 & 51145 & 5.997 & 22952 \\
\hline Avg & 1.152 & 3395917 & 4.590 & 50892 & 5.997 & 22787 \\
\hline Std Dev & 0.001 & 4419.56 & 0.004 & 604.81 & 0.001 & 123.91 \\
\hline RSD & 0.090 & 0.130 & 0.094 & 1.188 & 0.022 & 0.544 \\
\hline
\end{tabular}

Table: 5 Method Precision

\begin{tabular}{|c|c|c|c|c|c|c|}
\hline \multirow{2}{*}{ Name } & \multicolumn{2}{|c|}{ Metformin } & \multicolumn{2}{c|}{ Pioglitazone } & \multicolumn{2}{c|}{ Glibenclamide } \\
\cline { 2 - 7 } & RT & Area & RT & Area & RT & Area \\
\hline Method Precision-1 & 1.152 & 3395773 & 4.589 & 51145 & 5.997 & 22952 \\
\hline Method Precision-2 & 1.151 & 3395865 & 4.585 & 51246 & 5.998 & 22965 \\
\hline Method Precision-3 & 1.153 & 3396856 & 4.584 & 51151 & 5.998 & 22972 \\
\hline Method Precision-4 & 1.152 & 3396545 & 4.592 & 51344 & 5.997 & 22765 \\
\hline Method Precision-5 & 1.150 & 3396745 & 4.582 & 51145 & 5.995 & 22856 \\
\hline Method Precision-6 & 1.150 & 3397535 & 4.595 & 51421 & 5.996 & 22768 \\
\hline Avg & 1.151 & 3396553 & 4.588 & 51242 & 5.997 & 22880 \\
\hline Std Dev & 0.001 & 659.52 & 0.005 & 117.95 & 0.001 & 97.18 \\
\hline RSD & 0.105 & 0.019 & 0.110 & 0.230 & 0.019 & 0.425 \\
\hline
\end{tabular}


Table 6: Intraday Precision

\begin{tabular}{|c|c|c|c|c|c|c|}
\hline \multirow{2}{*}{ Name } & \multicolumn{2}{|c|}{ Metformin } & \multicolumn{2}{c|}{ Pioglitazone } & \multicolumn{2}{c|}{ Glibenclamide } \\
\cline { 2 - 7 } & RT & Area & RT & Area & RT & Area \\
\hline Injection -1 & 1.153 & 3390269 & 4.598 & 50264 & 5.999 & 22594 \\
\hline Injection-2 & 1.152 & 3391873 & 4.588 & 51645 & 5.997 & 22789 \\
\hline Injection-3 & 1.151 & 3402394 & 4.590 & 50611 & 5.996 & 22738 \\
\hline Injection-4 & 1.152 & 3396645 & 4.591 & 51444 & 5.997 & 22765 \\
\hline Injection-5 & 1.154 & 3398545 & 4.587 & 50244 & 5.995 & 22884 \\
\hline Injection-6 & 1.152 & 3395773 & 4.586 & 51145 & 5.997 & 22952 \\
\hline Avg & 1.152 & 3395917 & 4.590 & 50892 & 5.997 & 22787 \\
\hline Std Dev & 0.001 & 4419.56 & 0.004 & 604.81 & 0.001 & 123.91 \\
\hline RSD & 0.090 & 0.130 & 0.094 & 1.188 & 0.022 & 0.544 \\
\hline
\end{tabular}

Table 7: Interday Precision

\begin{tabular}{|c|c|c|c|c|c|c|}
\hline \multirow{2}{*}{ Name } & \multicolumn{2}{|c|}{ Metformin } & \multicolumn{2}{c|}{ Pioglitazone } & \multicolumn{2}{c|}{ Glibenclamide } \\
\cline { 2 - 7 } & RT & Area & RT & Area & RT & Area \\
\hline Injection-1 & 1.155 & 3380243 & 4.592 & 51455 & 5.996 & 22695 \\
\hline Injection -2 & 1.155 & 3391475 & 4.598 & 51262 & 5.992 & 22656 \\
\hline Injection -3 & 1.154 & 3372596 & 4.592 & 51173 & 5.998 & 22842 \\
\hline Injection -4 & 1.153 & 3396747 & 4.596 & 51264 & 5.985 & 22575 \\
\hline Injection -5 & 1.155 & 3388646 & 4.585 & 51358 & 5.991 & 22476 \\
\hline Injection -6 & 1.153 & 3395877 & 4.593 & 51472 & 5.988 & 22865 \\
\hline Avg & 1.154 & 3387597 & 4.593 & 51331 & 5.992 & 22685 \\
\hline Std Dev & 0.001 & 9460.78 & 0.004 & 118.49 & 0.005 & 150.84 \\
\hline RSD & 0.085 & 0.279 & 0.097 & 0.231 & 0.081 & 0.665 \\
\hline
\end{tabular}

\subsection{Robustness}

As defined by $\mathrm{ICH}$, the robustness of an analytical procedure describes to its capability toRemain unaffected by small and deliberate variations in method parameters. Robustness wasPerformed by small variation in the chromatographic conditions and found to be unaffected by small variations like flow rate $( \pm 10 \%)$, column oven temperature $\left( \pm 5^{\circ} \mathrm{c}\right)$ and wave length ( \pm 5 units). The values are summarized in Table 8 .

Table 8: Robustness

\begin{tabular}{|c|c|c|c|}
\hline Drug & Flow rate & Column oven temperature & Wavelength \\
\hline \multirow{2}{*}{ Metformin hydrochloride } & $0.7 \mathrm{ml}$ & $25^{\circ} \mathrm{c}$ & $249 \mathrm{~nm}$ \\
& $0.9 \mathrm{ml}$ & $35^{\circ} \mathrm{c}$ & $259 \mathrm{~nm}$ \\
\hline \multirow{2}{*}{ Pioglitazone hydrochloride } & $0.7 \mathrm{ml}$ & $25^{\circ} \mathrm{c}$ & $259 \mathrm{~nm}$ \\
\hline \multirow{2}{*}{ Glibenclamide } & $0.9 \mathrm{ml}$ & $35^{\circ} \mathrm{c}$ & $249 \mathrm{~nm}$ \\
& $0.7 \mathrm{ml}$ & $25^{\circ} \mathrm{c}$ & $259 \mathrm{~nm}$ \\
\hline
\end{tabular}

\subsection{Ruggedness}

The method is rugged by different analyst; different time intervals and the method did not significantly affect the recoveries, peak area and retention time of all the above drugs indicating that the proposed method is rugged. The values are summarized in Table 9.

Table 9: Ruggedness

\begin{tabular}{|c|c|c|c|c|c|c|}
\hline \multirow{2}{*}{ Name } & \multicolumn{2}{|c|}{ Metformin } & \multicolumn{2}{c|}{ Pioglitazone } & \multicolumn{2}{c|}{ Glibenclamide } \\
\cline { 2 - 7 } & RT & Area & RT & Area & RT & Area \\
\hline Injection -1 & 1.153 & 3390269 & 4.598 & 50264 & 5.999 & 22594 \\
\hline Injection-2 & 1.152 & 3391873 & 4.588 & 51645 & 5.997 & 22789 \\
\hline Injection -3 & 1.151 & 3402394 & 4.590 & 50611 & 5.996 & 22738 \\
\hline Injection-4 & 1.152 & 3396645 & 4.591 & 51444 & 5.997 & 22765 \\
\hline
\end{tabular}


Stability Indicating RP-HPLC Method for the Simultaneous Estimation of Metformin Hydrochloride,

\begin{tabular}{|c|c|c|c|c|c|c|}
\hline Injection -5 & 1.154 & 3398545 & 4.587 & 50244 & 5.995 & 22884 \\
\hline Injection -6 & 1.152 & 3395773 & 4.586 & 51145 & 5.997 & 22952 \\
\hline Avg & 1.152 & 3395917 & 4.590 & 50892 & 5.997 & 22787 \\
\hline Std Dev & 0.001 & 4419.56 & 0.004 & 604.81 & 0.001 & 123.91 \\
\hline RSD & 0.090 & 0.130 & 0.094 & 1.188 & 0.022 & 0.544 \\
\hline
\end{tabular}

Table 10: Validation Parameters of the Method

\begin{tabular}{|l|c|c|c|}
\hline \multicolumn{1}{|c|}{ Method Parameters } & Metformin & Pioglitazone & Glibenclamide \\
\hline Linearity range $(\mu \mathrm{g} / \mathrm{ml})$ & $62.5-375.00$ & $3.75-22.5$ & $1.25-7.50$ \\
\hline Correlation coefficient & 0.999 & 0.998 & 0.999 \\
\hline LOD $(\mathrm{ng} / \mathrm{ml})$ & 2.98 & 3.008 & 9.98 \\
\hline LOQ $(\mathrm{ng} / \mathrm{ml})$ & 9.94 & 9.886 & 5.996 \\
\hline Retention time & 1.15 & 4.578 & 5577 \\
\hline Theoretical plates & 2241 & 5041 & 1.01 \\
\hline Tailing factor & 1.15 & 1.05 & 0.544 \\
\hline Precision(\%RSD) & 0.130 & 1.188 & 0.022 \\
\hline Intra-day $(\mathrm{n}=3)$ & 0.09 & 0.094 & 0.081 \\
\hline Inter-day $(\mathrm{n}=3)$ & 0.085 & 0.097 & 99.98 \\
\hline$\%$ Recovery $(\mathrm{n}=6)$ & 100.08 & 100.14 & \\
\hline
\end{tabular}

\section{Forced Degradation Studies (Stress Testing)}

Forced degradation studies were carried out for all the three drugs. The bulk drugs were subjected to alkaline studies by adding $1.0 \mathrm{ml}$ of $0.1 \mathrm{M} \mathrm{NaOH}$ for $4 \mathrm{hrs}$, $8 \mathrm{hrs}$ and $12 \mathrm{hrs}$ neutralized with $1.0 \mathrm{ml}$ of $0.1 \mathrm{M} \mathrm{HCl}$ acid. Similarly, the acidic studies were performed by adding $1.0 \mathrm{ml}$ of $0.1 \mathrm{M} \mathrm{HCl}$ for $4 \mathrm{hrs}$, 8hrs and $12 \mathrm{hrs}$ and neutralized with $1 \mathrm{ml}$ of $0.1 \mathrm{M} \mathrm{NaOH}$. Oxidation studies were performed on bulk drug by adding $1.0 \mathrm{ml}$ of $3 \%$ $\mathrm{H} 2 \mathrm{O} 2$, thermalstudies were performed by keeping the drug at $100^{\circ} \mathrm{Cand} \mathrm{UV}$ studies were performed with UVLamp for $4 \mathrm{hrs}, 8 \mathrm{hrs}$ and $12 \mathrm{hrs}$ respectively (Figure10-14). All samples were taken in different $10 \mathrm{ml}$ volumetric flask and dissolved in mobile phase. Final assay drug concentration was made up with mobile phase and injected in the chromatographic system. For all the stability study, the formation of degradable product was confirmed by comparing to chromatogram of the solution kept under normal conditions. All stressed samples were analyzed by developed HPLC method. The degradation data for metformin hydrochloride, pioglitazone hydrochloride, glibenclamide was shown in Table 11, 12, 13 respectively.

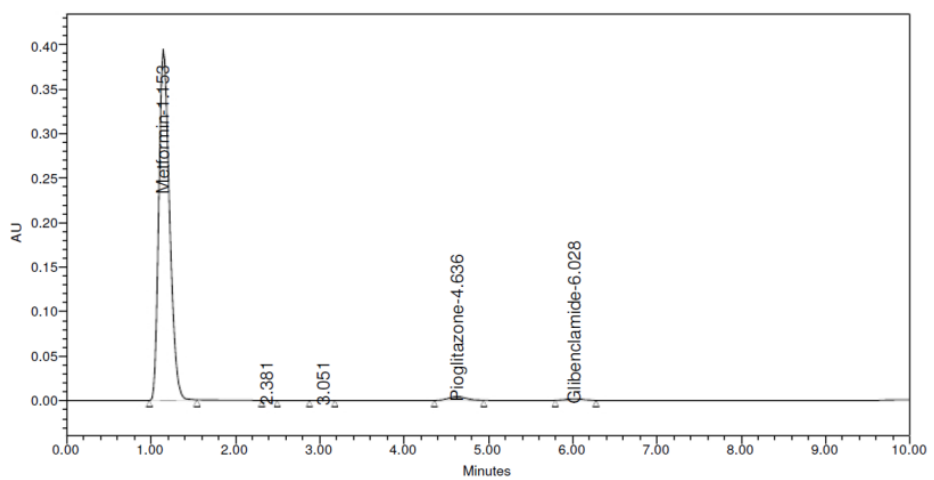

Fig. 10 Typical Chromatogram of Acid Hydrolysis

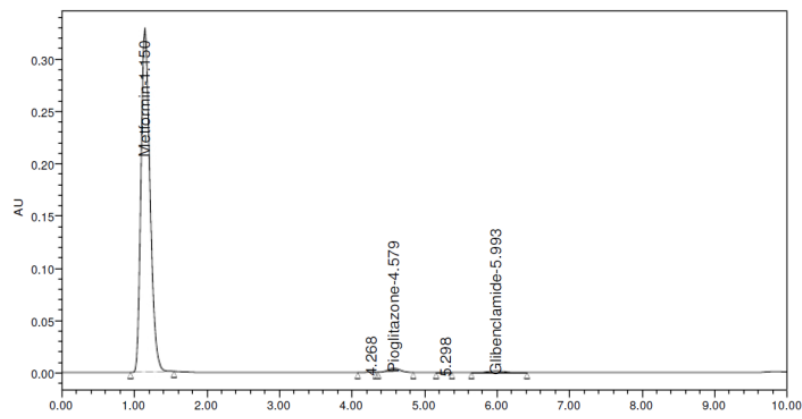

Fig. 11 Typical Chromatogram of Alkaline hydrolysis 


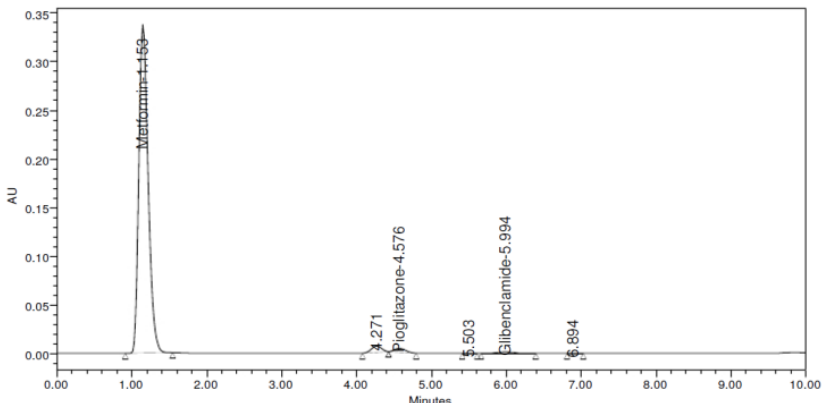

Fig. 12 Typical Chromatogram of Thermal Degradation

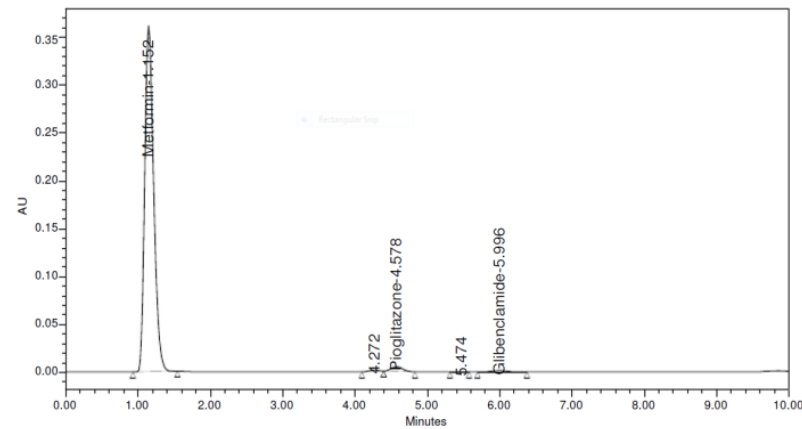

Fig. 13 Typical Chromatogram of UV Degradation

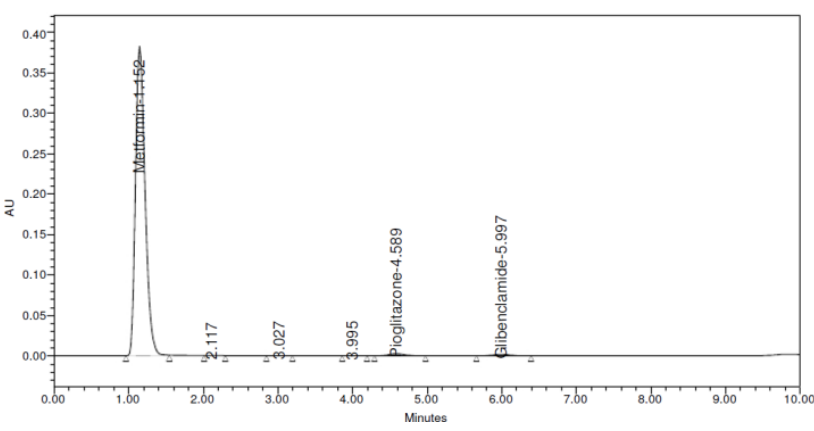

Fig. 14 Typical Chromatogram of Oxidation

Table 11: Degradation data for Metformin Hydrochloride

\begin{tabular}{|c|c|c|}
\hline Stress condition & Degradation time & Degradation (\%) \\
\hline \multirow{3}{*}{$\begin{array}{c}\text { Acidic } \\
(0.1 \mathrm{Hcl})\end{array}$} & $4 \mathrm{hrs}$ & 97.39 \\
\hline & $8 \mathrm{hrs}$ & 95.71 \\
\hline & $12 \mathrm{hrs}$ & 93.47 \\
\hline \multirow{3}{*}{$\begin{array}{l}\text { Alkaline } \\
(0.1 \mathrm{NaOH})\end{array}$} & $4 \mathrm{hrs}$ & 94.56 \\
\hline & $8 \mathrm{hrs}$ & 89.80 \\
\hline & $12 \mathrm{hrs}$ & 86.56 \\
\hline \multirow{3}{*}{$\begin{array}{c}\text { Oxidation } \\
\left(\mathrm{H}_{2} \mathrm{O}_{2}\right)\end{array}$} & 4hrs & 92.17 \\
\hline & $8 \mathrm{hrs}$ & 86.63 \\
\hline & $12 \mathrm{hrs}$ & 82.7 \\
\hline \multirow{3}{*}{ UV } & $4 \mathrm{hrs}$ & 96.99 \\
\hline & $8 \mathrm{hrs}$ & 94.48 \\
\hline & $12 \mathrm{hrs}$ & 91.52 \\
\hline \multirow{3}{*}{ Thermal } & $4 \mathrm{hrs}$ & 92.36 \\
\hline & $8 \mathrm{hrs}$ & 88.73 \\
\hline & $12 \mathrm{hrs}$ & 85.87 \\
\hline
\end{tabular}


Table 12: Degradation data for Pioglitazone Hydrochloride

\begin{tabular}{|c|c|c|}
\hline Stress condition & Degradation time & Degradation (\%) \\
\hline \multirow{2}{*}{$\begin{array}{c}\text { Acidic } \\
(0.1 \mathrm{Hcl})\end{array}$} & $4 \mathrm{hrs}$ & 99.01 \\
\cline { 2 - 3 } & $8 \mathrm{hrs}$ & 98.96 \\
\cline { 2 - 3 } & $12 \mathrm{hrs}$ & 98.04 \\
\hline \multirow{2}{*}{$\begin{array}{c}\text { Alkaline } \\
(0.1 \mathrm{NaOH})\end{array}$} & $4 \mathrm{hrs}$ & 95.12 \\
\cline { 2 - 3 } & $8 \mathrm{hrs}$ & 90.36 \\
\hline \multirow{2}{*}{$\begin{array}{c}\text { Oxidation } \\
\left(\mathrm{H}_{2} \mathrm{O}_{2}\right)\end{array}$} & $12 \mathrm{hrs}$ & 87.59 \\
\cline { 2 - 3 } & $4 \mathrm{hrs}$ & 93.05 \\
\cline { 2 - 3 } & $8 \mathrm{hrs}$ & 87.63 \\
\hline \multirow{3}{*}{ Thermal } & $12 \mathrm{hrs}$ & 82.91 \\
\cline { 2 - 3 } & $4 \mathrm{hrs}$ & 96.03 \\
\cline { 2 - 3 } & $8 \mathrm{hrs}$ & 91.48 \\
\hline \multirow{2}{*}{$\mathrm{UV}$} & $12 \mathrm{hrs}$ & 86.99 \\
\cline { 2 - 3 } & $4 \mathrm{hrs}$ & 99.41 \\
\cline { 2 - 3 } & $8 \mathrm{hrs}$ & 98.72 \\
\hline
\end{tabular}

Table 13: Degradation data for Glibenclamide

\begin{tabular}{|c|c|c|}
\hline Stress condition & Degradation time & Degradation (\%) \\
\hline \multirow{2}{*}{$\begin{array}{c}\text { Acidic } \\
(0.1 \mathrm{Hcl})\end{array}$} & $4 \mathrm{hrs}$ & 97.13 \\
\cline { 2 - 3 } & $8 \mathrm{hrs}$ & 95.89 \\
\cline { 2 - 3 } & $12 \mathrm{hrs}$ & 93.51 \\
\cline { 2 - 3 } $\begin{array}{c}\text { Alkaline } \\
(0.1 \mathrm{NaOH})\end{array}$ & $4 \mathrm{hrs}$ & 96.73 \\
\cline { 2 - 3 } & $8 \mathrm{hrs}$ & 94.27 \\
\hline \multirow{2}{*}{$\begin{array}{c}\text { Oxidation } \\
\left(\mathrm{H}_{2} \mathrm{O}_{2}\right)\end{array}$} & $12 \mathrm{hrs}$ & 91.42 \\
\cline { 2 - 3 } & $4 \mathrm{hrs}$ & 93.98 \\
\cline { 2 - 3 } & $8 \mathrm{hrs}$ & 87.13 \\
\cline { 2 - 3 } Thermal & $12 \mathrm{hrs}$ & 83.43 \\
\cline { 2 - 3 } & $4 \mathrm{hrs}$ & 92.37 \\
\hline \multirow{2}{*}{$\mathrm{UV}$} & $8 \mathrm{hrs}$ & 89.28 \\
\cline { 2 - 3 } & $12 \mathrm{hrs}$ & 86.86 \\
\cline { 2 - 3 } & $4 \mathrm{hrs}$ & 97.63 \\
\cline { 2 - 3 } & $8 \mathrm{hrs}$ & 95.17 \\
\hline
\end{tabular}

\section{Conclusion}

A novel, simple, rapid and cost effective RP-HPLC method was successfully developed for simultaneous determination of metformin hydrochloride, pioglitazone hydrochloride and glibenclamide. The proposed method was optimized and validated for the various experimental parameters. Influence of $\mathrm{pH}$ of the mobile phase, column oven temperature and various particulate columns on the analysis of metformin hydrochloride, pioglitazone hydrochloride and glibenclamide was evaluated. All the analytes were well resolved and separated in less than $10 \mathrm{~min}$. The developed method is a stability indicating method and can be conveniently used by quality control outfits to determine the contents of metformin hydrochloride, pioglitazone hydrochloride and glibenclamide simultaneously in routine and stability samples. This method could be used for the analysis of the drugs in pharmaceutical preparations and routine laboratory analysis with slight modification in the extraction procedure. Overall, the proposed method provides high throughput for simultaneous determination of metformin hydrochloride, pioglitazone hydrochloride and glibenclamide with excellent accuracy, precision, selectivity and reproducibility.

\section{Aknowledgement}

The authors express their sincere thanks to the Principal, JSS College of Pharmacy, Mysore and JSS University, Mysore for providing the facilities to carry out the research work.

\section{References}

[1] K.D Tripathi, Essentials of Medical Pharmacology, Jaypee Brothers Medical Publishers, New Delhi,2003, p.246.

[2] Chaturvedi RSPK(2008) Simultaneous spectrophotometric estimation and validation of three component tablet formulation containing pioglitazone hcl, metformin hcl and glibenclamide Analytical Letters 12:2133-2142

[3] Kishore L, Kaur N (2011) Estimation of pioglitazone and glimepiride in its pharmaceutical dosage form by spectrophotometric methods.Der Pharmacia Lettre 4:276-284

[4] Sane T, Menon N, Shafi I, Mandar M, Ajay M. Simultaneousdetermination of pioglitazone and glimepiride by high-performance thinlayerchromatography, J. Planar Chromatogr., 2004; 17: 154-156

[5] Zhong WZ, Lakings DB. Determination of pioglitazone in dog serum using solid-phase extraction and high-performance liquidchromatography with ultraviolet $(229 \mathrm{~nm})$ detection. J. Chromatogr. 1989; 490: 377-385. 
[6] P. Sripalakit, P. Neamhom, A. Saraphanchotiwitthaya, High- performance liquid chromatographic method for the determina- tion of pioglitazone in human plasma using ultraviolet detection and its application to a pharmacokinetic study, J. Chromatogr. B 843 (2006) 164-169

[7] Emilsson H, Sjöberg S, Svedner M, Christenson I (1986) High performance liquid chromatographic determination of glibenclamide in human plasma. J Chromatogr383: 93-102.

[8] M.A. Marques, A.S. Soares, O.W. Pinto, et al., Simple and rapid method determination for metformin in human plasma using high performance liquid chromatography tandem mass spectrometry: application to pharmacokinetic studies, J. Chromatogr. B 852 (2007) 308-316

[9] P. Sengupta, U. Bhaumik, A. Ghosh, et al., LC-MS-MS develop- ment and validation for simultaneous quantitation of metformin, glimepiride and pioglitazone in human plasma and its application to a bioequivalence study, Chromatographia 69 (2009) 12431250.

[10] S. AbuRuz, J. Millership, J. McElnay, The development and validation of liquid chromatography method for the simultaneous determination of metformin and glipizide, gliclazide, glibenclamide or glimperide in plasma, J. Chromatogr. B 817 (2005) $277-286$.

[11] ICH, Q2A Validation of Analytical Procedures: Consensus Guidelines; ICH Harmonized Tripartite Guidelines, 1994

[12] ICH, Q2B Validation of Analytical Procedures: Methodology, Consensus Guidelines; ICH Harmonized Tripartite Guidelines, 1996

[13] validation of analytical procedures, Proceedings of the International Conference onHarmonization (ICH). Commission of the Japan (1997). 